\title{
Energy-Per-Bit Performance of Relay-assisted Power Line Communication Systems
}

\author{
Khaled M. Rabie, Member, IEEE, Bamidele Adebisi, Senior Member, IEEE, Haris Gacanin, Senior IEEE, and \\ Serhan Yarkan, Member, IEEE
}

\begin{abstract}
This study provides a comprehensive analysis of the energy efficiency performance for different relaying schemes over the non-Gaussian power line communication (PLC) channel. Specifically, amplify-and-forward (AF), decode-and-forward (DF), selective DF (SDF) and incremental DF (IDF) relaying systems are investigated. For a more realistic scenario, the power consumption profile of the PLC modems is assumed to consist of both dynamic and static power. For each system, we derive accurate analytical expressions for the outage probability and the minimum energy-per-bit performance. For the sake of comparison and completeness as well as to quantify the achievable gains, we also analyze the performance of a single-hop PLC system. Monte Carlo simulations are provided throughout the paper to validate the theoretical analysis. Results reveal that AF relaying over the non-Gaussian PLC channel does not always enhance the performance and that the IDF PLC system offers the best performance compared to all other schemes considered. It is also shown that increasing the channel variance, which is related to the PLC network branching, and impulsive noise probability can considerably deteriorate the system performance. Furthermore, when the end-to-end distance is relatively small, it is found that the single-hop PLC approach can perform better than AF relaying.
\end{abstract}

Index Terms-Energy efficiency, impulsive noise, incremental DF, log-normal fading, selective DF, outage probability, power line communication (PLC).

\section{INTRODUCTION}

$\mathbf{T}$ HE power line communication (PLC) technology exploits the existing and pre-installed electric wires for data transmissions despite their unfavorable signal propagation at higher bands. The main advantage of PLC resides in the significant reduction of deployment costs and the elimination of hardto-reach access problem in buildings and power grid. PLCs promise many applications for both indoor and outdoor environments, including home-networking, control applications, smart metering etc [1]-[3]. In addition, future PLC systems are envisioned as an integral part of $5 \mathrm{G}$ networks to provide wireline backhaul for wireless access points. However, for reliable communications over these cables, many practical challenges must to be overcome such as the non-Gaussian noise, distance-dependent attenuation, frequency selectivity and electromagnetic compatibility (EMC) issues [4]-[6]. Multi-carrier

Khaled M. Rabie and Bamidele Adebisi are with the school of Electrical Engineering, Manchester Metropolitan University, Manchester, M15 6BH, UK. (e-mails: k.rabie@mmu.ac.uk; b.adebisi@mmu.ac.uk).

Haris Gacanin is a research director with Nokia, Copernicuslaan 50, 2018 Antwerp, Belgium. (e-mail: haris.gacanin@nokia.com).

Serhan Yarkan is the Director of the Center of Applied Research on Informatics Technologies (CARIT), Istanbul Commerce University, Istanbul 34840, Turkey (e-mail: syarkan@ticaret.edu.tr). modulation schemes such as orthogonal frequency-division multiplexing (OFDM) and relaying protocols (both cooperative and non-cooperative) are two effective strategies, able to marginalize the severity of the aforementioned challenges [7]-[10]. Over the past decade, numerous relaying protocols have been presented in the literature attempting to enhance the performance and quality of PLC links. The two main protocols are: amplify-and-forward (AF) and decode-and-forward (DF) [9]-[11]. It is shown that remarkable capacity improvements can be attained with the use of relays and that the PLC system reliability can be further improved by increasing the number of relaying modems in comparison to the direct-link transmission. However, increasing the number of relays increases the total power consumption due to the static power of each modem (i.e., the power consumption associated with the circuitry) [12].

In recent years, a number of studies have appeared in the literature investigating the issue of energy efficiency in relaying PLC systems [13]-[15]. Following this considerable research attention devoted to energy efficiency in PLCs in recent times, it is set to become a critical design criterion in future PLC networks. These studies considered different relaying and power consumption profiles of PLC modems to reduce transmit power. This is mainly achieved by optimizing the system parameters such as time slot allocation, relay position, etc. Unlike the previous work, the authors of [16], [17] proposed harvesting the unwanted high impulsive noise energy at the relay to further enhance the energy efficiency of relaying PLC systems. Several relaying and energy-harvesting protocols were investigated. For instance, in [16], [18] the authors considered an AF dual-hop relaying PLC network with time-switching relaying for energy harvesting. The study in [17] analyzed the energy efficiency performance of a DF dualhop relaying PLC system with time-switching relaying. In this work, the authors implement and discuss several frequency selection and power allocation strategies such as optimal frequency selection, random frequency selection and equal power allocation.

Despite all the work done on this topic, to the best of the authors' knowledge, a comprehensive and in-depth theoretical performance analysis of relaying PLC systems is still missing in the literature. This analysis will grant better understanding on system behavior in different scenarios which is necessary for efficient design of practical PLC systems. This paper is therefore dedicated to analyze the outage probability and energy efficiency of various relaying schemes over outdoor PLC channels. More specifically, we consider practically most rele- 
vant $\mathrm{AF}$, DF, selective DF (SDF) and incremental (IDF) transmission strategies. Note that the first two schemes are noncooperative whereas the last two are cooperative schemes. In order to highlight the achievable gains of each scheme as well as for completeness sake, we also analyze the performance of a single-hop PLC system, i.e, direct transmission without relay. Two performance metrics are adopted in this work, namely, the average outage probability and the energy efficiency in terms of the energy-per-bit performance. The main contribution of this paper resides in deriving accurate analytical expressions for the average outage probability and energy efficiency of the transmission strategies above. Computer simulations are presented to corroborate the theoretical analysis. The derived analytical expressions are used to assess and gain insights into the systems performance and design in different environments as well as to make comparisons between the performance of the different systems under consideration.

The results reveal that AF relaying over the non-Gaussian PLC channel does not always enhance performance compared to the single-hop system, specially when the noise is very impulsive and/or when the end-to-end distance is relatively small. It is also presented that the IDF PLC scheme offers the best performance in comparison to the other schemes under study followed by the SDF PLC approach. When the energy processing cost of the DF relay is not taken into account, the AF PLC system has slightly worse energy efficiency performance relative to the DF PLC one which indicates that the former scheme might still be attractive in applications where complexity is the main parameter in system design. Finally, it is found that increasing the impulsive noise probability and/or its amplitude, the channel variance (which is related to the network branching) will considerably degrade the performance ${ }^{1}$.

The rest of the paper is organized as follows. Section II presents the system model. Section III analyzes the average outage probability and energy-per-bit performance of the single-hop, AF, DF, SDF and IDF relaying systems. Numerical examples and simulation results are discussed in Section IV. Finally, Section V concludes the paper.

\section{System MOdEL}

The relaying PLC systems considered in this study consist of a source modem (S), a relaying modem (R) and a destination modem (D). The source-to-relay, relay-to-destination and source-to-destination channel coefficients are complex values with log-normally distributed amplitudes [20], denoted as $h_{1}$, $h_{2}$ and $h_{0}$, respectively, with the corresponding distances being $d_{1}, d_{2}$ and $d_{0}=d_{1}+d_{2}$. The means and standard deviations of $10 \log _{10}\left(h_{m}\right)$ are denoted as $\mu_{m}$ and $\sigma_{m}$, respectively, where $m \in\{0,1,2\}$. Hence, the probability density function (PDF) of the channel coefficients can be written as

$$
f\left(z_{m}\right)=\frac{\zeta}{\sqrt{2 \pi} \sigma z_{m}} \exp \left[-\frac{\left(10 \log _{10}\left(z_{m}\right)-\mu_{m}\right)^{2}}{2 \sigma_{m}^{2}}\right],
$$

${ }^{1}$ Note that part of this paper was presented at the IEEE ICC 2017 [19]. where $z_{m}=h_{m}^{2}$ and $\zeta=10 / \ln (10)$ is a scaling constant.

The cable losses are modeled as $A\left(f, d_{m}\right)=\exp \left(-\alpha d_{m}\right)$, where $\alpha=a_{o}+a_{1} f^{k}$ is the attenuation factor, $f$ is the operating frequency, $k$ is the exponent of the attenuation factor, $d_{m}$ is the distance, and $a_{o}$ and $a_{1}$ are constants determined from measurements which depend on the network topology and structure [4], [21], [22].

Five transmission strategies are studied, namely, single-hop, $\mathrm{AF}$ relaying, DF relaying, SDF relaying and IDF relaying. Note that AF and DF are non-cooperative schemes since the destination modem can only received and decode the relayed signal from the relay whereas SDF and IDF are referred to as cooperative schemes because they are able to also exploit the direct signal from the source modem along with the relayed one. The energy efficiency in this study is calculated as the transmit energy-per-bit, which is basically the total transmit power over the data rate. The adopted power consumption profile in our analysis takes into account not only the dynamic power but also the static power of the PLC modems consumed by the circuitry; for more details, the reader may refer to [14], [23].

Furthermore, to accurately characterize the PLC channel impairments, the noise at all modems is assumed to consist of both background noise $\left(n_{w}\right)$ with variance $\sigma_{w}^{2}$ and impulsive noise $\left(n_{i}\right)$ with variance $\sigma_{i}^{2}$. The well-known GaussianBernoulli noise model is used to characterize these noise components in which the probability occurrence of impulsive noise is represented by $p$ [24]. The variances $\sigma_{w}^{2}$ and $\sigma_{i}^{2}$ define the signal-to-background noise ratio (SBNR) and the signal-to-impulsive noise ratio (SINR), respectively, as $\mathrm{SBNR}=10 \log _{10}\left(1 / \sigma_{w}^{2}\right)$ and $\operatorname{SINR}=10 \log _{10}\left(1 / \sigma_{i}^{2}\right)$. Note that for ease of notation and without loss of generality, the noise characteristics at all modems are assumed to be identical.

\section{Performance Analysis}

In this section, we analyze the average outage probability and energy efficiency performances of the transmission schemes discussed above.

\section{A. Single-hop PLC System}

In the single-hop approach, the source communicates directly with the destination modem. In this case, the energyper-bit performance can be calculated as

$$
E_{b, \mathrm{SH}}=\frac{P_{\mathrm{SH}}^{*}+P_{s t c}^{T x}+P_{s t c}^{R x}}{R_{b}},
$$

where $E_{b, \mathrm{SH}}$ is the energy/bit of the single-hop system, $P_{\mathrm{SH}}^{*}$ is the optimal transmit power for a given outage probability, i.e., the minimum transmit power to achieve a certain outage probability, $R_{b}=\xi B$ is the data rate in bits/s, $\xi$ is the spectral efficiency, $B$ is the system bandwidth in $\mathrm{Hz}, P_{s t c}^{T x}$ and $P_{s t c}^{R x}$ are the static powers of the transmitting and receiving modems, respectively. It should be highlighted that the static power is circuitry-specific; therefore, to minimize the overall energy consumption, the transmit power must be minimized. 
We now derive the average outage probability for this system. To begin with, the received signal at the destination modem in the single-hop case can be written as

$$
y_{D}=\sqrt{P_{\mathrm{SH}}} A\left(f, d_{0}\right) h_{0} x+n_{w}+n_{i},
$$

where $P_{\mathrm{SH}}$ is the source transmit power and $x$ is the source information signal.

The SBNR at the destination can simply be expressed as

$$
\gamma_{D}=\frac{P_{\mathrm{SH}} A\left(f, d_{0}\right)^{2} h_{0}^{2}}{\sigma_{w}^{2}} .
$$

In impulsive noise channels, such as the power line channel, the outage probability can be determined as follows [25], [26]

$$
P_{\text {out }}^{\mathrm{SD}}=\operatorname{Pr}\left\{\sum_{i=0}^{1} p_{i} \log _{2}\left(1+\gamma_{D, i}\right)<\xi\right\},
$$

where the superscript $\{\mathrm{SD}\}$ denotes the source-to-destination link, $p_{0}=1-p, p_{1}=p, \gamma_{D, 0}=\gamma_{D}, \gamma_{D, 1}=\gamma_{D} / \beta$ and $\beta=1+\sigma_{i}^{2} / \sigma_{w}^{2}$.

To simplify our analysis, we use the high signal-to-noise ratio (SNR) approximation. In light of this, we can reduce (5) to

$$
P_{\text {out }}^{\mathrm{SD}} \simeq \operatorname{Pr}\left\{\left(\gamma_{D}\right)^{1-p}\left(\frac{\gamma_{D}}{\beta}\right)^{p}<2^{\xi}\right\}
$$

which, with some basic mathematical manipulations, can also be written as

$$
\begin{aligned}
P_{\text {out }}^{\text {SD }} & \simeq \operatorname{Pr}\left\{\log _{2}\left(\gamma_{D}\right)^{1-p}+\log _{2}\left(\frac{\gamma_{D}}{\beta}\right)^{p}<\xi\right\} \\
& \simeq \operatorname{Pr}\left\{\left(\gamma_{D}\right)^{1-p}+\left(\frac{\gamma_{D}}{\beta}\right)^{p}<2^{\xi}\right\} \\
& \simeq \operatorname{Pr}\left\{\gamma_{D}<\beta^{p} 2^{\xi}\right\} .
\end{aligned}
$$

It is clear that the expression in (7) basically represents the cumulative distribution function (CDF) of $\gamma_{D}$. Hence, (7) can be expressed as

$$
P_{\text {out }}^{\mathrm{SD}} \simeq F_{\gamma_{D}}\left(\beta^{p} 2^{\xi}\right)
$$

where $F_{\gamma_{D}}(\cdot)$ denotes the CDF of $\gamma_{D}$.

From (4), one can see that $\gamma_{D}$ has log-normal distribution because $h_{0}^{2}$ is log-normally distributed. Consequently, and using the properties of log-normal distribution, the average outage probability $P_{\text {out }}^{\mathrm{SD}}$ can now be written as

$$
P_{\mathrm{out}}^{\mathrm{SD}} \simeq \frac{1}{2}+\frac{1}{2} \operatorname{erf}\left(\frac{\zeta \ln \left(\beta^{p} 2^{\xi}\right)-\left(2 \mu_{0}+\zeta \ln \left[\Xi_{0}\right]\right)}{\sqrt{8} \sigma_{0}}\right),
$$

where $\Xi_{0}=P_{\mathrm{SH}} A\left(f, d_{0}\right) / \sigma_{w}^{2}$.
Now, fixing the average outage probability at the limit $P_{\text {out }}^{*}$ and using (9), it is easy to show that the minimum transmit power that can achieve this performance is

$$
P_{\mathrm{SH}}^{*}=\frac{\beta^{p} 2^{\xi} \sigma_{w}^{2}}{A\left(f, d_{0}\right)} \exp \left(-\frac{\sqrt{8} \sigma_{0} \operatorname{erf}^{-1}\left(2 P_{\mathrm{out}}^{*}-1\right)+2 \mu_{0}}{\zeta}\right) .
$$

Finally, substituting (10) into (2) yields the energy-per-bit performance of the single-hop PLC system.

\section{B. Amplify-and-Forward (AF) Relaying PLC System}

This section focuses on the performance of AF PLC systems. The energy-per-bit performance of this scheme is given by

$$
E_{b, \mathrm{AF}}=\frac{2 P_{\mathrm{AF}}^{*}+2 P_{s t c}^{T x}+2 P_{s t c}^{R x}}{N R_{b}}
$$

where $E_{b, \mathrm{AF}}$ is the energy/bit of the AF relaying system, $P_{\mathrm{AF}}^{*}$ is the optimal transmit power for a given outage probability. It is worthwhile noting that a parameter $N$ has now appeared in the denominator of (11). Note that $N=2$ for all the relaying schemes considered from now onward because in dual-hop relaying, the overall throughput is reduced to half since the end-to-end communication is accomplished over two time slots, instead of one in the single-hop scheme.

The overall outage probability of this system is obtained as follows. The end-to-end communication requires two time slots. In the first time slot, the source modem transmits its signal to the relay where the received signal can be written as

$$
y_{R}=\sqrt{P_{s, \mathrm{AF}}} A\left(f, d_{1}\right) h_{1} x+n_{w}+n_{i},
$$

where $P_{s, \mathrm{AF}}$ is the source transmit power.

In the second time slot, the relay amplifies and forwards the source message to the destination; hence, the received signal at the destination can be given by

$$
\begin{aligned}
y_{D}= & \sqrt{P_{s, \mathrm{AF}} P_{r, \mathrm{AF}}} A\left(f, d_{1}\right) A\left(f, d_{2}\right) G h_{1} h_{2} x \\
& +\sqrt{P_{r, \mathrm{AF}}} h_{2} A\left(f, d_{2}\right) G n_{r}+n_{d},
\end{aligned}
$$

where $P_{r, \mathrm{AF}}$ is the relay transmit power, $G$ is the relay gain and $n_{r}$ and $n_{d}$ are the total noises at the relay and destination modems, respectively.

From (13), and substituting $X=h_{1}^{2}$ and $Y=h_{2}^{2}$, the SBNR at the destination can be expressed as

$$
\gamma_{D}=\frac{P_{s, \mathrm{AF}} P_{r, \mathrm{AF}} A\left(f, d_{1}\right)^{2} A\left(f, d_{2}\right)^{2} X Y G^{2}}{P_{r, \mathrm{AF}} Y A\left(f, d_{2}\right)^{2} G^{2} \sigma_{r}^{2}+\sigma_{w}^{2}} .
$$

The end-to-end outage probability of this system can be given as 


$$
P_{\mathrm{out}}^{\mathrm{AF}}=\operatorname{Pr}\left\{\frac{1}{2} \sum_{i=0}^{1} p_{i} \log _{2}\left(1+\gamma_{D, i}\right)<\xi\right\},
$$

where $\gamma_{D, 0}=\gamma_{D}$ and $\gamma_{D, 1}=\gamma_{D} / \beta$.

Using the high SNR approximation, we can express (15) as

$$
P_{\mathrm{out}}^{\mathrm{AF}} \simeq \operatorname{Pr}\left\{\gamma_{D}<\beta^{p} 2^{2 \xi}\right\}
$$

Now, using (14) (16), we can write the outage probability as follows

$$
P_{\mathrm{out}}^{\mathrm{AF}} \simeq \operatorname{Pr}\left\{\frac{P_{s, \mathrm{AF}} P_{r, \mathrm{AF}} A\left(f, d_{1}\right)^{2} A\left(f, d_{2}\right)^{2} X Y G^{2}}{P_{r, \mathrm{AF}} Y A\left(f, d_{2}\right)^{2} G^{2} \sigma_{r}^{2}+\sigma_{w}^{2}}<\beta^{p} 2^{2 \xi}\right\}
$$

Using the substitutions $a=P_{s, \mathrm{AF}} P_{r, \mathrm{AF}} A\left(f, d_{1}\right)^{2}$ $A\left(f, d_{2}\right)^{2} G^{2}, b=\sigma_{w}^{2}$ and $c=P_{r, \mathrm{AF}} A\left(f, d_{2}\right)^{2} G^{2} \sigma_{r}^{2}$, we can now rewrite (17) as

$$
P_{\text {out }}^{\mathrm{AF}} \simeq \operatorname{Pr}\left\{\frac{a X Y}{c Y+b}<\beta^{p} 2^{2 \xi}\right\},
$$

which can also be expressed as

$$
P_{\text {out }}^{\mathrm{AF}} \simeq \operatorname{Pr}\left\{Y<\frac{b \beta^{p} 2^{2 \xi}}{a X-c \beta^{p} 2^{2 \xi}}\right\} .
$$

The probability in (19) can be mathematically calculated as

$$
P_{\mathrm{out}}^{\mathrm{AF}}=\int_{0}^{\frac{c \beta^{p_{2} 2 \xi}}{a}} f_{X}(z) \mathrm{d} z+\int_{\frac{c \beta^{p} 2^{2 \xi}}{a}}^{\infty} f_{X}(z) F_{Y}(z) \mathrm{d} z
$$

where $f_{X}(\cdot)$ is the PDF of $X$ and $F_{Y}(z)$ is the CDF of $Y$.

Note that the random variables (RVs) $X$ and $Y$ are log-normally distributed with parameters $L N\left(2 \mu_{1}, 4 \sigma_{1}^{2}\right)$ and $L N\left(2 \mu_{2}, 4 \sigma_{2}^{2}\right)$, respectively. With this in mind, we can express $f_{X}(\cdot)$ and $F_{Y}(z)$ respectively as follows

$$
f_{X}(z)=\frac{\zeta}{z \sqrt{8 \pi \sigma_{1}^{2}}} \exp \left(\frac{\zeta \ln (z)-2 \mu_{1}}{8 \sigma_{1}^{2}}\right),
$$

and

$$
F_{Y}(z)=1-Q\left(\frac{\zeta \ln (\Gamma)-2 \mu_{2}}{2 \sigma_{2}}\right)
$$

where $\Gamma=\frac{b \beta^{p} 2^{2 \xi}}{a z-c \beta^{p} 2^{2 \xi}}$ and $Q(\cdot)$ is the Q-function defined as

$$
Q(x)=\frac{1}{\sqrt{2 \pi}} \int_{x}^{\infty} \exp \left(-\frac{u^{2}}{2}\right) \mathrm{d} u
$$

Substituting (21) and (22) into (20), and with some algebraic manipulations, we can calculate the average outage probability of the AF-based system as

$$
\begin{aligned}
P_{\mathrm{out}}^{\mathrm{AF}}=1- & \frac{\zeta}{\sqrt{8 \pi \sigma_{1}^{2}}} \int_{\frac{c \beta^{p} 2 \xi}{a}}^{\infty} \exp \left(\frac{\zeta \ln (z)-2 \mu_{1}}{8 \sigma_{1}^{2}}\right) \\
& \times \frac{1}{z} Q\left(\frac{\zeta \ln (\Gamma)-2 \mu_{2}}{2 \sigma_{2}}\right) \mathrm{d} z .
\end{aligned}
$$

Note that although it is challenging to express the average outage probability (24) in closed-form, a numerical solution can be easily obtained using software tools. Now, fixing the outage probability $P_{\text {out }}^{\mathrm{AF}}$ in (24) at $P_{\text {out }}^{*}$, and using software tools (specifically the function FindRoot in Mathematica was used), we can obtain numerical results for the minimum transmit power. Finally, substituting these values into (11), we obtain the energy-per-bit performance of the AF PLC system.

\section{Decode-and-Forward Relaying PLC System}

The relay in this system decodes the received source signal and then forwards it to the destination. The total energy-per-bit consumption in this case is calculated as

$$
E_{b, \mathrm{DF}}=\frac{P_{\mathrm{DF}}^{*}+P_{s t c}^{T x}+P_{s t c}^{R x}}{N R_{b}}\left(P_{\mathrm{out}}^{\mathrm{SR}}+2\left(1-P_{\mathrm{out}}^{\mathrm{SR}}\right)\right),
$$

where $E_{b, \mathrm{DF}}$ is the energy/bit of the DF relaying scheme, $P_{\text {out }}^{\mathrm{SR}}$ denotes the outage probability of the source-to-relay link and $P_{\mathrm{DF}}^{*}$ is the optimal transmit power for a given outage probability of the overall system. Similar to the AF relaying approach, the parameter $N$ appears in the denominator of (25). Note that the first term in (25) represents the energy consumption when the decoding at the relay is unsuccessful, i.e., when the data is lost, whereas the second terms denotes the energy consumption when the decoding is successful, i.e., when the data is successfully forwarded to the destination modem.

To calculate the energy efficiency of this system, we first derive the overall outage probability. Assuming that the relay is placed midway between the source and destination modems, the end-to-end outage probability of this system can be given by

$$
P_{\text {out }}^{\mathrm{DF}}=P_{\text {out }}^{\mathrm{SR}}+\left(1-P_{\mathrm{out}}^{\mathrm{SR}}\right) P_{\text {out }}^{\mathrm{RD}},
$$

where $P_{\text {out }}^{\mathrm{RD}}$ is the outage probability of the relay-to-destination link.

Following the same steps as in Sec. III-A, and, without loss of generality, assuming that the source and relay have same transmit power of $P_{\mathrm{DF}}$, the outage probabilities $P_{\text {out }}^{\mathrm{SR}}$ and $P_{\mathrm{out}}^{\mathrm{RD}}$ can be respectively expressed as

$$
P_{\text {out }}^{\mathrm{SR}} \simeq 1-\mathrm{Q}\left(\frac{\zeta \ln \left(\beta^{p} 2^{\xi}\right)-\left(2 \mu_{1}+\zeta \ln \left[\Xi_{1}\right]\right)}{2 \sigma_{1}}\right),
$$




$$
P_{\text {out }}^{\mathrm{RD}} \simeq 1-\mathrm{Q}\left(\frac{\zeta \ln \left(\beta^{p} 2^{\xi}\right)-\left(2 \mu_{2}+\zeta \ln \left[\Xi_{2}\right]\right)}{2 \sigma_{2}}\right),
$$

where $\Xi_{1}=P_{\mathrm{DF}} A\left(f, d_{1}\right) / \sigma_{w}^{2}$ and $\Xi_{2}=P_{\mathrm{DF}} A\left(f, d_{2}\right) / \sigma_{w}^{2}$.

Note that when the source-to-relay and relay-to-destination links are identical in terms of fading statistics and distances, i.e., $\mu=\mu_{1}=\mu_{2}, \sigma^{2}=\sigma_{1}^{2}=\sigma_{2}^{2}$, and $d=d_{1}=d_{2}$, the average outage probability can be expressed as

$P_{\mathrm{out}}^{\mathrm{DF}}=1-Q\left(\frac{\zeta \ln \left(\sigma_{w}^{2} \beta^{p} 2^{2 \xi}\right)-2 \mu-\zeta \ln \left(A(f, d) P_{D F}\right)}{2 \sigma}\right)^{2}$.

Now, substituting (27) and (28) into (26) while replacing $P_{\text {out }}^{\text {DF }}$ with $P_{\text {out }}^{*}$, we can numerically find the optimal transmit power $\left(P_{\mathrm{DF}}^{*}\right)$ for a given outage probability $P_{\mathrm{out}}^{*}$. Finally, substituting the resultant $P_{\mathrm{DF}}^{*}$ into (25), we obtain the consumed energy-per-bit for the DF relaying PLC system.

\section{Selective DF Relaying PLC System}

In this approach, the source transmits its information signal to the destination during the first time slot which can also be heard by the relaying modem. In the second time slot, the relay will decode and forward the received signal to the destination. The destination then performs selection combining based on the two received copies from the source and relay during the two time slots. The relay in this approach will always cooperate as long as it successfully decodes the received signal, unlike the IDF scheme as will be discussed later.

With this in mind, we can write the total consumed energyper-bit of this system as follows

$$
\begin{aligned}
E_{b, \mathrm{SDF}}= & P_{\mathrm{out}}^{\mathrm{SR}} \frac{P_{\mathrm{SDF}}^{*}+P_{s t c}^{T x}+2 P_{s t c}^{R x}}{N R_{b}} \\
& +\left(1-P_{\mathrm{out}}^{\mathrm{SR}}\right) \frac{2 P_{\mathrm{SDF}}^{*}+2 P_{s t c}^{T x}+3 P_{s t c}^{R x}}{N R_{b}},
\end{aligned}
$$

where $E_{b, \mathrm{SDF}}$ is the energy/bit in this system, $P_{\mathrm{SDF}}^{*}$ is the optimal transmit power for a given overall outage probability which can be calculated for this case as

$$
P_{\mathrm{out}}^{\mathrm{SDF}}=P_{\mathrm{out}}^{\mathrm{SD}}\left(P_{\mathrm{out}}^{\mathrm{SR}}+\left(1-P_{\mathrm{out}}^{\mathrm{SR}}\right) P_{\mathrm{out}}^{\mathrm{RD}}\right) .
$$

Note that the additional $P_{s t c}^{R x}$ in (30) is due to the fact that the destination will be decoding the source signal at the same time as the relay, during the first time. The probabilities $P_{\mathrm{out}}^{\mathrm{SD}}$, $P_{\text {out }}^{\mathrm{SR}}$ and $P_{\text {out }}^{\mathrm{SD}}$ in (31) can be obtained from (9), (27) and (28), respectively, by replacing $P_{\mathrm{SH}}$ and $P_{\mathrm{DF}}$ with $P_{\mathrm{SDF}}$.

Now, replacing $P_{\text {out }}^{\mathrm{SD}}$ in (31) with $P_{\text {out }}^{*}$, we can numerically find the corresponding optimal transmit power $P_{\mathrm{SDF}}^{*}$ which is then substituted into (30) to obtain the overall energy-per-bit performance of the SDF PLC system.
Note that when the source-to-relay and relay-to-destination links are identical, the average outage probability of the SDF system can be simplified to

$$
\begin{aligned}
P_{\mathrm{out}}^{\mathrm{SDF}} & =\left(1-Q\left(\frac{\zeta \ln (\Phi)-2 \mu_{0}-\zeta \ln \left(\triangle_{0}\right)}{2 \sigma_{0}}\right)\right) \\
& \times\left(1-Q\left(\frac{\zeta \ln (\Phi)-2 \mu-\zeta \ln (\triangle)}{2 \sigma}\right)^{2}\right)
\end{aligned}
$$

where $\triangle_{0}=A\left(f, d_{0}\right) P_{\mathrm{SDF}}, \triangle=A(f, d) P_{\mathrm{SDF}}$ and $\Phi=$ $\sigma_{w}^{2} \beta^{p} 2^{2 \xi}$.

\section{E. Incremental DF Relaying PLC System}

The relay in the IDF protocol will only cooperate if requested to do so by the destination modem. To achieve this, a feedback channel is assumed to exist between the relay and destination. The availability of this feedback channel in this protocol allows the relay to avoid unnecessary data transmission; hence, consuming less power and considerably improving the energy efficiency. This protocol is based on the fact that relaying will only take place when the direct-link is unable to provide sufficient link quality [27]. Under this assumption, the energy consumed per bit can be written as

$$
\begin{aligned}
E_{b, \mathrm{IDF}}= & \left(1-P_{\mathrm{out}}^{\mathrm{SD}}\right) \frac{P_{\mathrm{IDF}}^{*}+P_{s t c}^{T x}+2 P_{s t c}^{R x}}{L R_{b}} \\
& +P_{\mathrm{out}}^{\mathrm{SD}} P_{\text {out }}^{\mathrm{SR}} \frac{P_{\mathrm{IDF}}^{*}+P_{s t c}^{T x}+2 P_{s t c}^{R x}}{R_{b}} \\
& +P_{\mathrm{out}}^{\mathrm{SD}}\left(1-P_{\mathrm{out}}^{\mathrm{SR}}\right) \frac{2 P_{\mathrm{IDF}}^{*}+2 P_{s t c}^{T x}+3 P_{s t c}^{R x}}{L R_{b}}
\end{aligned}
$$

where $E_{b, \mathrm{IDF}}$ is the energy/bit of the IDF system, $P_{\mathrm{IDF}}^{*}$ is the optimal transmit power for a given value of the endto-end outage probability. The first term in (33) indicates the energy consumed when the destination modem correctly decodes the source signal during the first time slot. The second term however refers to the energy consumption when none of the modems can correctly decode the source signal. The third component represents the energy consumption when cooperation occurs.

The average outage probability of the IDF scheme is equal to that of the SDF system; therefore, it can be easily obtained from (31) by making the appropriate notation changes. Following the same procedure as in the previous section, it is straightforward to find the energy-per-bit for the IDF relaying PLC system. This is omitted here for the sake of brevity.

\section{NumericAl Results}

In this section, we present and discuss some numerical examples of the average outage probability and energy efficiency expressions derived above. To validate these expressions, we provide Monte Carlo simulations based on $10^{6}$ iterations. Throughout this section, if not specified otherwise, we use $a_{0}=9.4 \times 10^{-3}, a_{1}=4.2 \times 10^{-7}, k=0.7, f=30 \mathrm{MHz}$, $\mathrm{SBNR}=25 \mathrm{~dB}, \mathrm{SINR}=-15 \mathrm{~dB}, p=0.01, P_{\text {out }}^{*}=0.01$, 


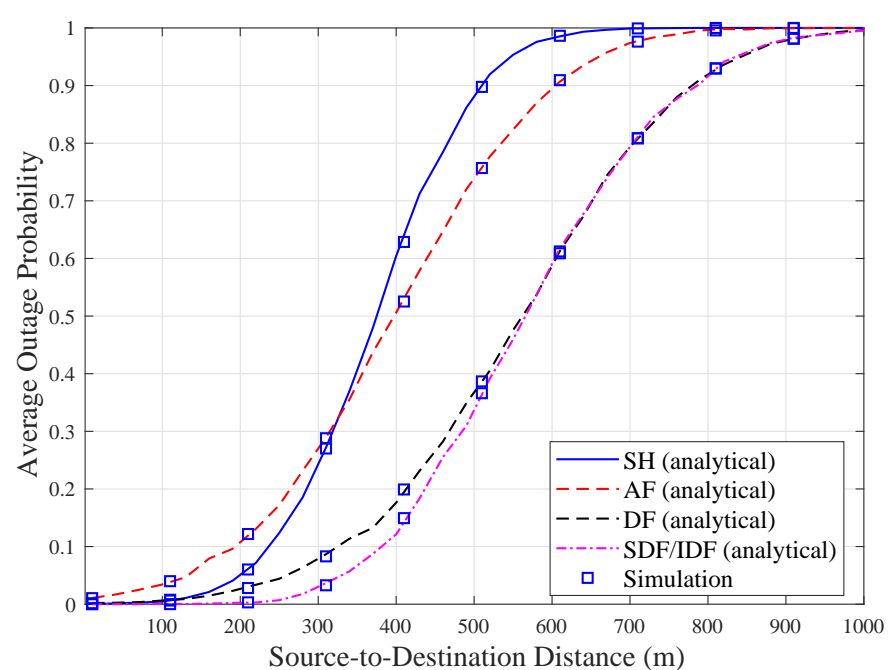

(a) $\mathrm{SINR}=-10 \mathrm{~dB}$ and $p=0.001$.

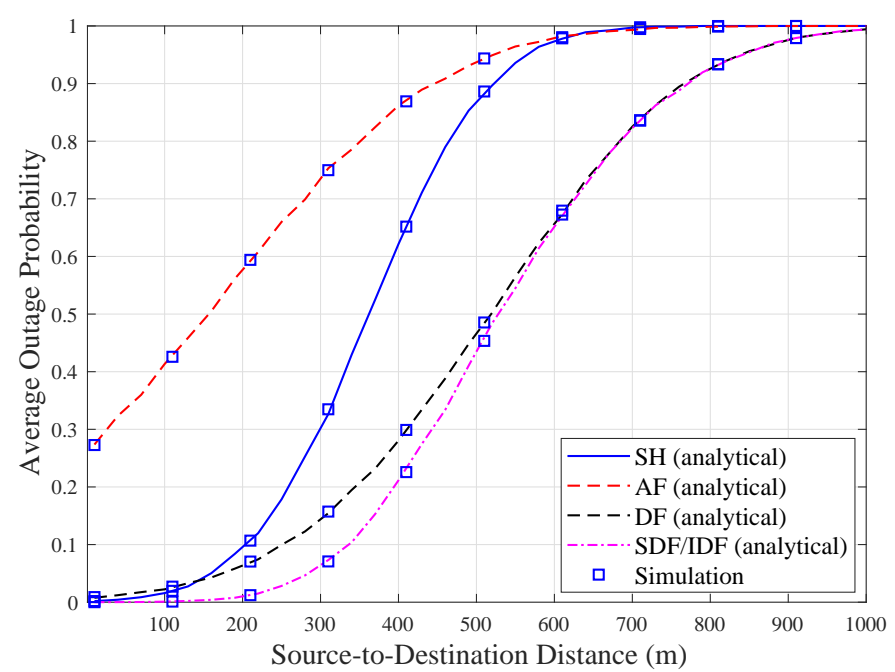

(b) SINR $=-15 \mathrm{~dB}$ and $p=0.01$.

Figure 1: Analytical and simulation results of the average outage probability performance with respect to the source-to-destination distance for the single-hop (SH), AF, DF and SDF/IDF relaying PLC systems with different noise scenarios.

$\mu_{m}=1 \mathrm{~dB}, \sigma_{m}^{2}=2 \mathrm{~dB}$, where $m \in\{0,1,2\}$. In addition, we assume that the relaying PLC modem is placed at the midpoint between the end modems.

\section{A. Average Outage Probability}

In this section, we discuss the average outage probability performance of the systems under consideration in various noise scenarios and system configurations. To start with, Fig. 1 illustrates some numerical examples of the outage probability with respect to the source-to-destination distance for the single-hop (SH), AF, DF and SDF/IDF relaying systems with two different impulsive noise scenarios, specifically $\{$ SINR $=$ $-10 \mathrm{~dB}, p=0.001\}$ and $\{\mathrm{SINR}=-15 \mathrm{~dB}, p=0.01\}$. The good agreement between the analytical and simulated curves clearly indicates the accuracy of our theoretical analysis. It is worth noting that the analytical results for the single-hop, AF, DF, SDF/IDF systems are obtained using (9), (24), (26) and (31), respectively. It is clear from both Figs. 1(a) and 1(b) that the SDF and IDF relaying systems always have the best performance compared to the other schemes irrespective of the noise characteristics and source-to-destination distance. The other common observation in the two figures is that the DF systems outperform the AF approach due to the noise propagation issue associated with AF relaying. In addition, it is noticeable that all systems perform poorly when the endto-end distance becomes very large (above $900 \mathrm{~m}$ ), which is intuitive. In such scenarios, more relays are required [12].

Having a closer look at the two figures, it can be observed that as the distance becomes larger, the performance of the SDF/IDF systems approaches that of the DF scheme and the gain gap becomes more pronounced as the noise power becomes higher. Furthermore, when the impulsive noise is less sever, i.e., Fig. 1(a), the single-hop system performs better than the AF-based scheme when the source-to-destination distance is relatively small, i.e., $\lesssim 300 \mathrm{~m}$ whereas when the distance becomes sufficiently large, i.e., $\gtrsim 300 \mathrm{~m}$, the advantage of relaying becomes obvious and the $\mathrm{AF}$ system achieves better outage probability. On the other hand, when the impulsive noise power is high, i.e., Fig. 1(b), the single-hop system can offer better outage probability performance compared to AF relaying throughout the distance considered. This can be justified as follows. When the noise becomes more impulsive and its power increases, the impact of noise propagation due to AF relaying becomes more severe resulting in significantly deteriorated performance at the destination modem. Hence, single-hop transmission becomes a more attractive solution under such conditions.

In order to assess the influence of the relay position on the system performance, we present in Fig. 2 the average outage probability as a function of the normalized sourceto-relay distance for the DF relaying systems when $d_{\mathrm{sd}}=$ $500 \mathrm{~m}, 700 \mathrm{~m}$ and $1000 \mathrm{~m}$. The first observation one can notice from these results is that, for all systems, the outage probability is minimized when the relay is placed exactly at the midpoint between the end modems and that the performance degrades as the relay is moved towards either the source and destination. It is also apparent that the SDF/IDF systems have considerably smaller outage probability in comparison to that of DF relaying and this performance enhancement becomes higher as the distance becomes smaller. Another result worth highlighting on this figure is that increasing the source-todestination distance always deteriorates performance. The last set of results in this section is shown in Fig. 3 which illustrates the impact of the SBNR on the system performance. As expected, clearly increasing the SBNR leads to a considerable performance improvement for all the DF relaying systems. It is also noticeable that the gain obtained with the SDF/IDF systems relative to the DF approach becomes higher as the SBNR increases. 


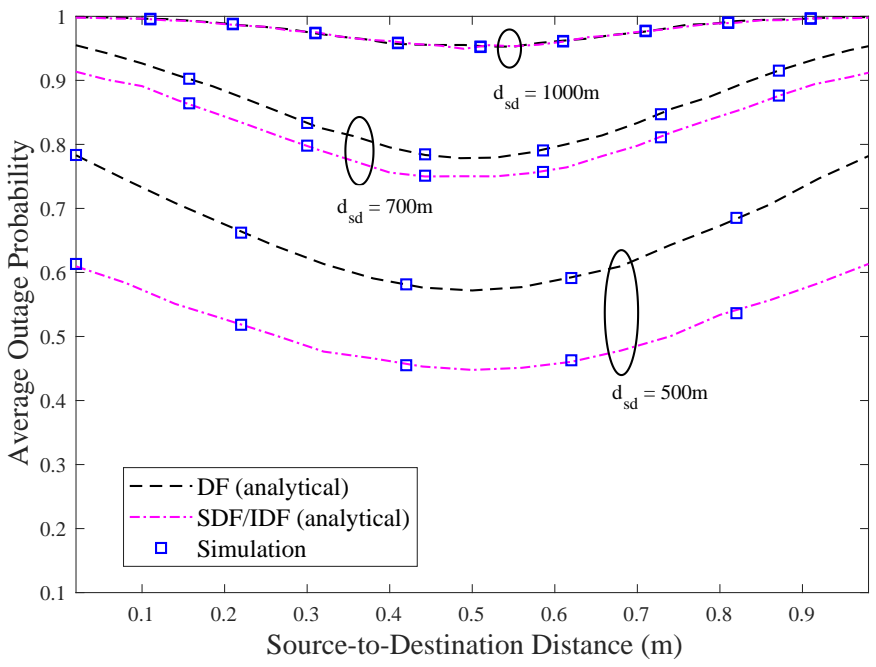

Figure 2: Average outage probability performance versus the normalized source-to-relay distance for the DF and SDF/IDF systems with several end-to-end distances.

\section{B. Energy-Per-Bit Performance}

This section focuses on evaluating the energy-per-bit performance of the single-hop, AF, DF, SDF and IDF relaying systems. Several system parameters are investigated and their impact on the energy-per-bit performance is illustrated in Fig. 4. This figure shows four sets of results for different system configurations; more specifically, it considers the following scenarios: $\left\{\sigma_{m}^{2}=3 \mathrm{~dB}, P_{\text {out }}^{*}=0.0001, \xi=1\right\},\left\{\sigma_{m}^{2}=3 \mathrm{~dB}\right.$, $\left.P_{\text {out }}^{*}=0.0001, \xi=3\right\},\left\{\sigma_{m}^{2}=3 \mathrm{~dB}, P_{\text {out }}^{*}=0.01, \xi=1\right\}$ and $\left\{\sigma_{m}^{2}=3.5 \mathrm{~dB}, P_{\text {out }}^{*}=0.0001, \xi=1\right\}$. All the results in this section, unless we explicitly specify otherwise, are based on the following: $p=0.001$, SINR $=-10 \mathrm{~dB}, \mathrm{SBNR}=25 \mathrm{~dB}$ and $\mu_{m}=4 \mathrm{~dB}$, where $m \in\{0,1,2\}$.

It is clear from Fig. 4 that, in all scenarios, the IDF relaying system is always the most energy efficient followed by the SDF scheme. This is because of the fact that in the former scheme, the relay will only cooperate if requested to do so by the destination modem when the signal from the direct link does not mean the requirement which eventually saves energy. Another observation one can notice is that, despite its ease of implementation in practice, AF relaying has higher energy-perbit consumption compared to the DF relaying approach due to the noise propagation issue discussed briefly in the previous section. Furthermore, it is interesting to note that the singlehop approach surpasses AF and DF relaying when the endto-end distance is relatively small. This indicates that the use of conventional AF and DF relaying can be energy-inefficient when the end modems are not distant enough. This is because of the increased static power caused by the increased number of modems. On the other hand, when the distance is large, the advantage of using relays becomes more visible.

Comparing Figs. 4(a) and 4(b), it can be noticed that increasing $\xi$ will always require higher energy-per-bit in all the systems. When the outage probability threshold is smaller, less energy will be used to meet the system performance; this

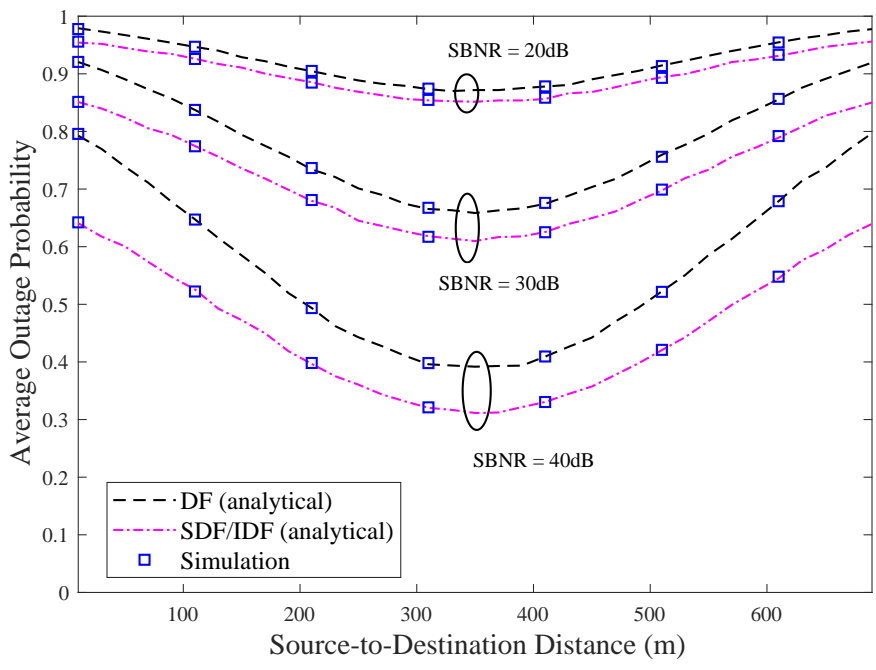

Figure 3: Average outage probability with respect to the source-todestination distance for the DF and SDF/IDF systems with several values of the SBNR plots. Note that the end-to-end distance is set to $700 \mathrm{~m}$.

is clear by comparing the results in Figs 4(a) and 4(c). It can also be observed by comparing Fig 4(a) and 4(d) that increasing the channel variance from $3 \mathrm{~dB}$ to $3.5 \mathrm{~dB}$ while keeping other system parameters unvaried will considerably increase the energy-per-bit consumption for all systems. This can be justified as follows. The PLC channel variance is affected by the power line network topology and its value increases as we increase the number of branches between the transmitting and receiving modems [25], [28]. Consequently, the channel becomes harsher and the required energy-per-bit to achieve a given outage probability will be higher resulting in higher energy consumption.

\section{CONCLuSions}

In this paper, we analyzed the average outage probability and energy-per-bit performance of the single-hop, AF, DF, SDF and IDF relaying PLC systems when the channel experiences log-normal fading and impulsive noise. Analytical expressions of the average outage probability and energyper-bit for the aforementioned schemes were derived and verified with computer simulations. These expressions have enabled us to make useful comparisons and reach interesting and insightful conclusions. For instance, it was shown that cooperative relaying, i.e., SDF and IDF, can provide better outage probability and energy efficiency performance in comparison to single-hop and non-cooperative $\mathrm{AF}$ and DF systems. Results also indicated that AF relaying does not always enhance the system performance over the nonGaussian PLC channel and its effectiveness is dependent on the impulsive noise characteristics. In fact, if the noise is very impulsive, AF relaying may worsen the system performance compared to the single-hop approach. It was also demonstrated that increasing the channel variance, i.e., the network branches, and the spectral efficiency threshold will increase the energyper-bit required to obtain the same performance. 


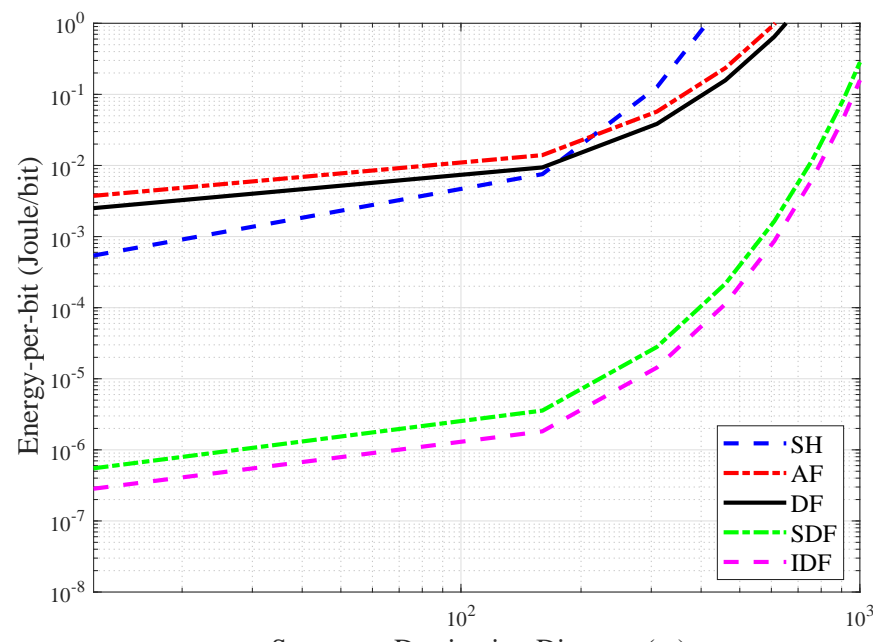

Source-to-Destination Distance (m)

(a) $\sigma_{m}^{2}=3 \mathrm{~dB}, P_{\text {out }}^{*}=0.0001$ and $\xi=1$.

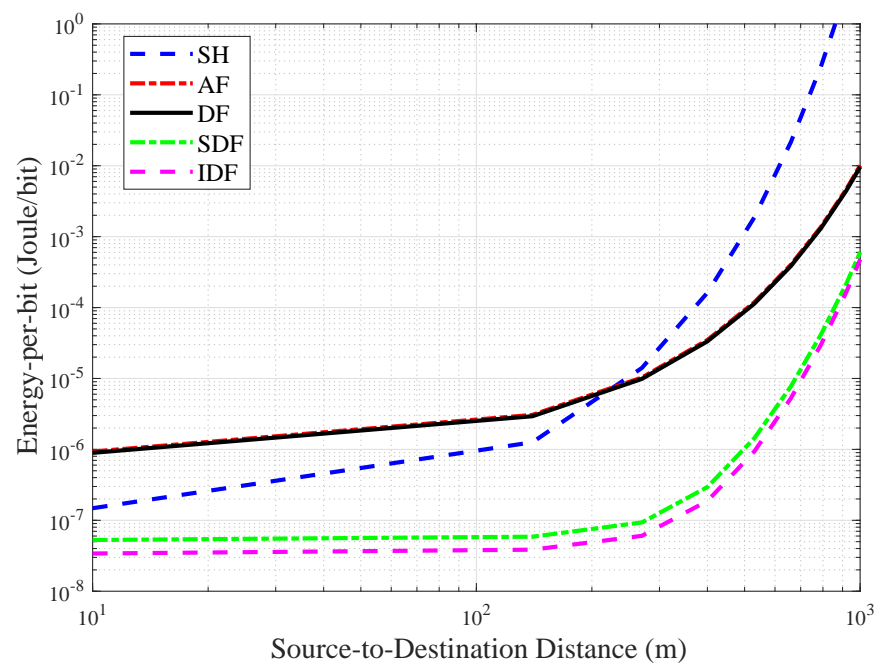

(c) $\sigma_{m}^{2}=3 \mathrm{~dB}, P_{\text {out }}^{*}=0.01$ and $\xi=1$.

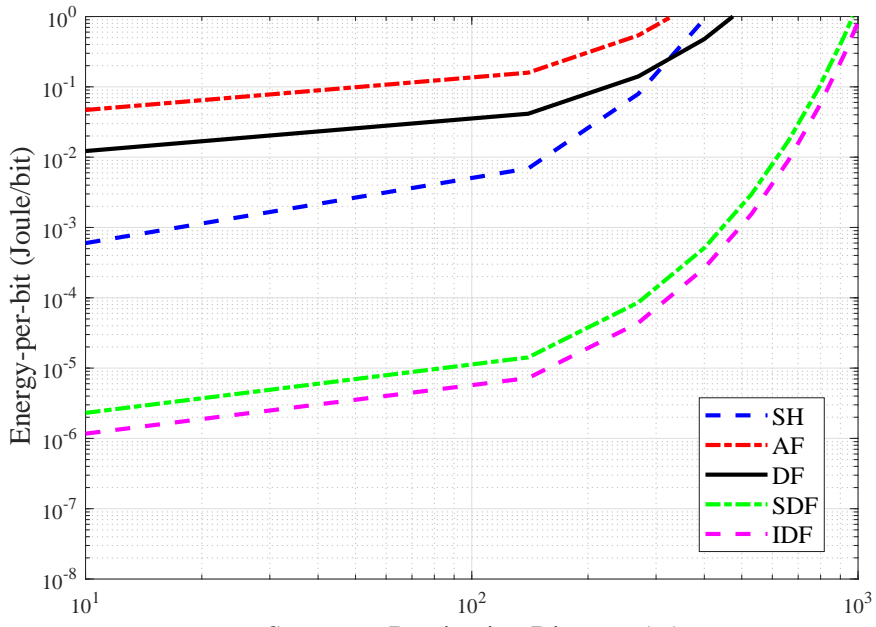

Source-to-Destination Distance (m)

(b) $\sigma_{m}^{2}=3 \mathrm{~dB}, P_{\text {out }}^{*}=0.0001$ and $\xi=3$.

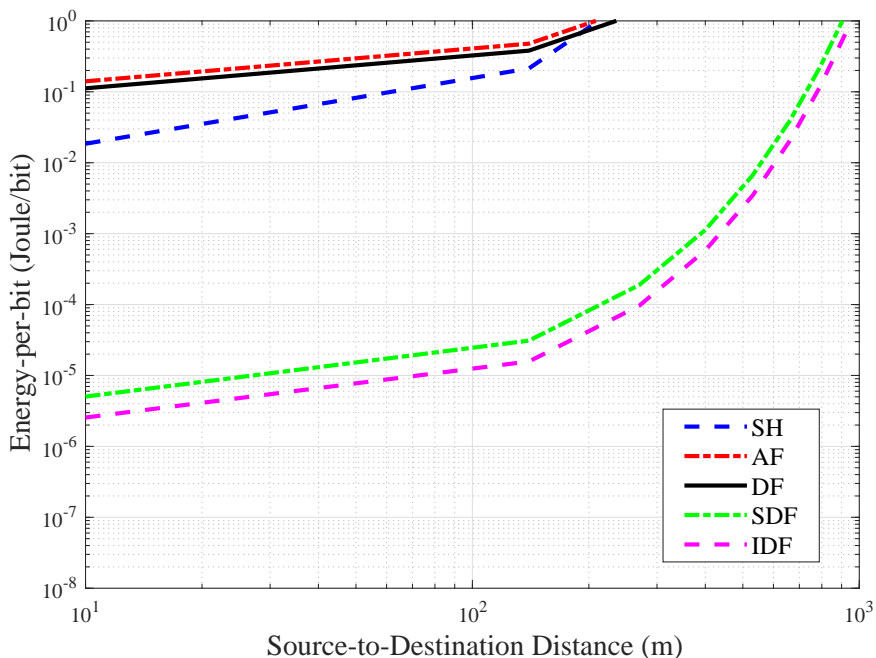

(d) $\sigma_{m}^{2}=3.5 \mathrm{~dB}, P_{\text {out }}^{*}=0.0001$ and $\xi=1$.

Figure 4: The energy-per-bit performance as a function of the source-to-destination distance for the single-hop, AF, DF, SDF and IDF PLC systems with several different system configurations.

\section{ACKNOWLEDGMENT}

This research has been carried out within the "CityVerve: IoTs and Smart Cities Demonstrator" project funded by Innovate UK (Project reference: 102561).

\section{REFERENCES}

[1] S. Galli, A. Scaglione, and Z. Wang, "For the grid and through the grid: The role of power line communications in the smart grid," Proc. IEEE, vol. 99, pp. 998-1027, Sept. 2011.

[2] A. Ikpehai, B. Adebisi, K. M. Rabie, R. Haggar, and M. Baker, "Experimental study of 6LoPLC for home energy management systems," Energies, vol. 9, no. 12, p. 1046, 2016.

[3] M. Nassar, J. Lin, Y. Mortazavi, A. Dabak, I. H. Kim, and B. Evans, "Local utility power line communications in the 3-500 khz band: Channel impairments, noise, and standards," IEEE Signal Process. Mag., vol. 29, pp. 116-127, Sept. 2012.

[4] M. Zimmermann and K. Dostert, "A multipath model for the powerline channel," IEEE Trans. Commun., vol. 50, pp. 553-559, Apr. 2002.

[5] B. Adebisi, J. Stott, and B. Honary, "Experimental study of the interference caused by PLC transmission on HF bands," in IET Int. Conf. Ionospheric Radio Systems Techniques (IRST), pp. 326-330, Jul. 2006.
[6] M. Rozman, A. Ikpehai, B. Adebisi, and K. M. Rabie, "Channel characterisation of cooperative relaying power line communication systems," in Proc. IEEE Int. Symp. Commun. Systems, Networks and Digital Signal Processing (CSNDSP), pp. 1-5, Jul. 2016.

[7] HomePlug AV White Paper. HomePlug Powerline Alliance, 2005.

[8] E. S. Hassan, "Multi user MIMO-OFDM-based power line communication structure with hardware impairments and crosstalk," IET Commun., vol. 11, no. 9, pp. 1466-1476, 2017.

[9] L. Lampe and A. Vinck, "Cooperative multihop power line communications," in Proc. IEEE Int. Symp. Power Line Commun. and Its Appl. (ISPLC), pp. 1-6, Mar. 2012.

[10] A. Tonello, F. Versolatto, and S. D'Alessandro, "Opportunistic relaying in in-home PLC networks," in Proc. IEEE Global Commun. Conf. (GLOBECOM), pp. 1-5, Dec. 2010.

[11] X. Cheng, R. Cao, and L. Yang, "Relay-aided amplify-and-forward powerline communications," IEEE Trans. Smart Grid, vol. 4, pp. 265272, Mar. 2013.

[12] K. M. Rabie, B. Adebisi, H. Gacanin, G. Nauryzbayev, and A. Ikpehai, "Performance evaluation of multi-hop relaying over non-Gaussian PLC channels," J. Commun. Networks, vol. 19, pp. 531-538, Oct. 2017.

[13] S. D'Alessandro and A. M. Tonello, "On rate improvements and power saving with opportunistic relaying in home power line networks," EURASIP J. Advances Signal Process., vol. 2012, pp. 1-17, Sept. 2012.

[14] W. Bakkali, P. Pagani, and T. Chonavel, "Energy efficiency performance 
of relay-assisted power-line communication networks," in Proc. IEEE Consumer Commun. and Networking Conf. (CCNC), pp. 525-530, Jan. 2015.

[15] W. Bakkali, P. Pagani, T. Chonavel, and A. M. Tonello, "Energy efficiency performance of decode and forward MIMO relay PLC systems," in Proc. IEEE Int. Symp. Power Line Commun. (ISPLC), pp. 201-205, Mar. 2016.

[16] K. M. Rabie, B. Adebisi, and A. Salem, "Improving energy efficiency in dual-hop cooperative PLC relaying systems," in Proc. IEEE Int. Symp. Power Line Commun. and Its Appl. (ISPLC), pp. 196-200, Mar. 2016.

[17] K. M. Rabie, B. Adebisi, A. M. Tonello, and G. Nauryzbayev, "For more energy efficient dual-hop DF relaying power line communication systems," IEEE Systems Journal, Jan. 2017.

[18] K. M. Rabie and B. Adebisi, "Enhanced amplify-and-forward relaying in non-gaussian PLC networks," IEEE Access, vol. 5, pp. 4087-4094, 2017.

[19] K. M. Rabie, B. Adebisi, and H. Gacanin, "Outage probability and energy efficiency of DF relaying power line communication networks: Cooperative and non-cooperative," in Proc. IEEE Int. Conf. Commun. (ICC), pp. 1-6, May 2017.

[20] A. M. Tonello, F. Versolatto, and A. Pittolo, "In-home power line communication channel: Statistical characterization," IEEE Trans. Commun., vol. 62, pp. 2096-2106, Jun. 2014

[21] K. Khalil, M. G. Gazalet, P. Corlay, F. X. Coudoux, and M. Gharbi, "An MIMO random channel generator for indoor power-line communication,” IEEE Trans. Power Del., vol. 29, pp. 1561-1568, Aug. 2014.

[22] J. Song, W. Ding, F. Yang, H. Yang, B. Yu, and H. Zhang, "An indoor broadband broadcasting system based on PLC and VLC," IEEE Trans. Broadcasting, vol. 61, pp. 299-308, Jun. 2015.

[23] W. Bakkali, M. Tlich, P. Pagani, and T. Chonavel, "A measurementbased model of energy consumption for PLC modems," in Proc. IEEE Int. Symp. Power Line Commun. and Its Appl. (ISPLC), pp. 42-46, Mar. 2014.

[24] M. Ghosh, "Analysis of the effect of impulse noise on multicarrier and single carrier QAM systems," IEEE Trans. Commun., vol. 44, pp. 145147, Feb. 1996.

[25] A. Dubey and R. K. Mallik, "PLC system performance with AF relaying," IEEE Trans. Commun., vol. 63, pp. 2337-2345, Jun. 2015.

[26] A. Dubey, R. K. Mallik, and R. Schober, "Performance analysis of a multi-hop power line communication system over log-normal fading in presence of impulsive noise," IET Commun., vol. 9, no. 1, pp. 1-9, 2015.

[27] M. T. Kakitani, G. Brante, R. D. Souza, and A. Munaretto, "Comparing the energy efficiency of single-hop, multi-hop and incremental decode-

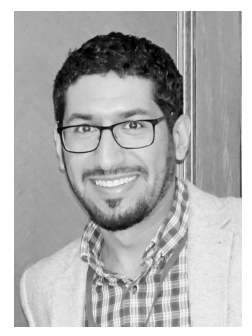

Khaled M. Rabie (S'12, M'15), received the B.Sc. degree (with Hons.) in Electrical and Electronic Engineering from the University of Tripoli, Tripoli, Libya, in 2008, and the M.Sc. and Ph.D. degrees in Communication Engineering both from the University of Manchester, Manchester, U.K., in 2010 and 2015, respectively. He is currently a Research Associate at Manchester Metropolitan University (MMU), Manchester, UK. His research interests include signal processing and analysis of power-line and wireless communication networks. He received several awards including the Agilent Technologies' best M.Sc. Student Award, the Manchester Doctoral College Ph.D. scholarship and the MMU Outstanding Knowledge Exchange Project award of 2016. He was also the recipient of the best student paper award at the IEEE International Symposium on Power Line Communications and its applications (ISPLC) in 2015, Texas, US. Khaled is the Publication Chair of the upcoming 2018 IEEE ISPLC, the IEEE CSNDSP Co-chair of the "Green Communications and Networks" track and an Editor of the Elsevier Physical Communication Journal. He is a Fellow of the UK Higher Academy of Education. and-forward in multi-relay wireless sensor networks," in Proc. IEEE Int. Symp. Personal, Indoor Mobile Radio Commun. (PIMRC), pp. 970-974, Sept. 2011.

[28] S. Guzelgoz, H. B. Celebi, and H. Arslan, "Statistical characterization of the paths in multipath PLC channels," IEEE Trans. Power Del., vol. 26, pp. 181-187, Jan. 2011.

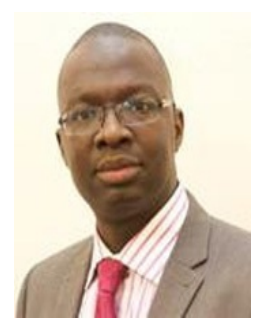

Bamidele Adebisi (M'06, SM'15) received his Master's degree in advanced mobile communication engineering and Ph.D. in communication systems from Lancaster University, UK, in 2003 and 2009 , respectively. Before that, he obtained a Bachelor's degree in electrical engineering from Ahmadu Bello University, Zaria, Nigeria, in 1999. He was a senior research associate in the School of Computing and Communication, Lancaster University between 2005 and 2012. He joined Metropolitan University, Manchester in 2012 where he is currently a Reader in Electrical and Electronic Engineering. He has worked on several commercial and government projects focusing on various aspects of wireline and wireless communications. He is particularly interested in Research and Development of communication technologies for electrical energy monitoring/management, transport, water, critical infrastructures protection, home automation, IoTs and Cyber Physical Systems. He has several publications and a patent in the research area of data communications over power line networks and smart grid. He is a member of IET and a senior member of IEEE.

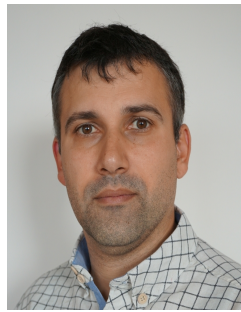

Haris Gacanin received his Dipl.-Ing. degree in Electrical engineering from the University of Sarajevo in 2000. In 2005 and 2008, respectively, he received $\mathrm{MSc}$ and $\mathrm{PhD}$ from Tohoku University in Japan. He was with Tohoku University from 2008 until 2010 first as Japan Society for Promotion of Science postdoctoral fellow and later, as Assistant Professor. In 2010, he joined Alcatel-Lucent (now Nokia), where he currently leads artificial intelligence communication systems research department within Nokia Bell Labs. His professional interests are related to application of artificial intelligence in autonomous networking, and design of mobile and wireless systems. He has $180+$ scientific publications (journals, conferences and patent applications) and invited/tutorial talks. He is senior member of the Institute of Electrical and Electronics Engineers (IEEE) and the Institute of Electronics, Information and Communication Engineering (IEICE).

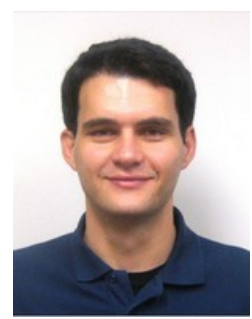

Serhan Yarkan received the B.S. and M.Sc. degrees in computer science from Istanbul University, Istanbul, Turkey, in 2001 and 2003, respectively, and the Ph.D. degree from the University of South Florida, Tampa, FL, USA, in 2009. He was a PostDoctoral Research Associate with the Department of Computer and Electrical Engineering, Texas AM University, College Station, TX, USA, from 2010 to 2012. Currently, he is an Associate Professor with the Department of Electrical-Electronics Engineering, Istanbul Commerce University, Istanbul. Serhan Yarkan's current research interests include statistical signal processing, cognitive radio, wireless propagation channel measurement and modeling, cross-layer adaptation and optimisation, and interference management in nextgeneration wireless networks and underground mine channels and disaster communications. 